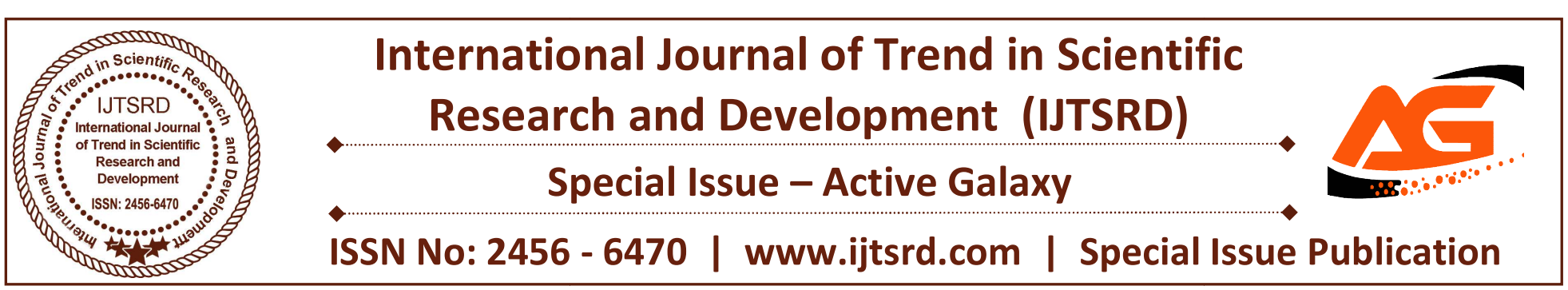

\title{
Effective Bandwidth Assurance in Data Center
}

\author{
Mr. Ganesh. T ${ }^{1}$, Dr. Nirmal kumar. A ${ }^{2}$, M. Gurubala ${ }^{3}$ \\ ${ }^{1}$ Assistant Professor, ${ }^{2}$ Associate Professor, ${ }^{2} \mathrm{PG}$ Student \\ ${ }^{1,2}$ Department of IT, ${ }^{2}$ Department of MCA, \\ ${ }^{1,2,3}$ Vel Tech High Tech Dr. Rangarajan Dr. Sakunthala Engineering College, \\ Chennai, Tamilnadu, India
}

\begin{abstract}
Designing a distributed algorithmic rule to attain an efficient and truthful bandwidth allocation. The bandwidth that isn't used by a network can even be shared proportionately between the active networks. This document examines accessible bandwidth measurement techniques and performs similar analyses in terms of accuracy, intrusion and interval. The transmission intervals of the data packets are accommodated within the ImTCP transmitter, that the accessible bandwidth of the network path between the sender and receiver is calculable by interleaving the arrival intervals of the ACK packets. It is represented that the queue prevents demanding information measure allocation algorithms. eBA an efficient bandwidth allocation, provision that has bandwidth for virtual machines with massive amounts of short streams and mass traffic in data centers and also the use of DBA(Dynamic bandwidth Allocation) with acceptable native queue management to mitigate this behavior, begin a new device to actively measure the bandwidth accessible on a network path. In recent years, many tools are designed to estimate network capability and accessible bandwidth. We have a tendency to use markov chain algorithms based on the calculation of the time between the arrivals of data packets.
\end{abstract}

KEYWORDS: Bandwidth, network path, virtual machines, accessible bandwidth, short streams

\section{INTRODUCTION}

The bandwidth between two virtual machine is significantly affected by traffic, leading to unexpected completion times of the activities. Bandwidth distribution among the various competitive demands becomes a key drawback in computer networks. The measurement mechanism shows mannerism of data center traffic. However, coming up with a Markov chain algorithm to manage the dynamic nature of data center traffic is harder than merely meeting basic requirements. Therefore, a wise allocation of bandwidth should not entirely meet the essential requirements, but also dynamically adapt to traffic patterns. Short periods of congestion are common in many connections. We have a tendency to point out, that the rule not only achieves bandwidth guarantees and assignments that retain work, but also adapts to extremely variable traffic in data centers. We tend to completely review the underlying design principles, evaluate design decisions and summarize them during a unified style space. The main reason of not accurately calculating the bandwidth of the highspeed network is that they do not take into account the capabilities of the host activity system. The network bandwidth estimation algorithm should not rely upon the performance of the ultimate host. The goal of a network measurement is to measure the available bandwidth of the network path, not the available bandwidth of the measurement host.

$$
\text { Bandwidth }=\frac{\text { size of requested image }}{\text { download time }} \ldots . .
$$

\section{LITERATURE SURVEY}

[1] J. Lee et al., proposed "Application-driven bandwidth guarantees in datacenters". Several tenants share the information center resource and their applications compete against one another because of the low bandwidth of the network. Current studies have shown that the lack of bandwidth guarantee causes unpredictable network performance, leading to 
poor application performance. To handle this drawback, many abstractions of virtual networks are projected that enable tenants to order virtual clusters with specified bandwidth between virtual machines (VMs) within the data centers. During this paper, we tend to explore a virtual cluster abstraction with stochastic bandwidth characterization to handle the uncertainty of bandwidth demand. We tend to propose the stochastic Virtual Cluster (SVC) that models the bandwidth demand among virtual machines in a very probabilistic method. Based on SVC, we have developed a stochastic framework for the allocation of virtual clusters, inside that the bandwidth requests of the supported virtual cluster are met with a high probability. Efficient VM allocation algorithms are projected to implement the framework whereas reducing the likelihood of connecting congestion by minimizing the utmost bandwidth occupation of a virtual cluster in physical links.

[2] L. Popa et al., proposed "Elastic Switch: practical work-conserving bandwidth guarantees for cloud computing". Data centers should offer tenants with bandwidth guarantees to predict performance. Ideally, this method should achieve three vital characteristics: job retention, fairness and simplicity. The first indicates that tenants will effectively use unused bandwidths while not damaging the bandwidth guarantee. The second indicates that tenants share the unused bandwidth when a given stock policy. The latter needs that the developed theme may be simply implemented with software and hardware of basic product. However, meeting all necessities at an equivalent time may be a challenge considering the distributed nature of bandwidth usage in data centers. In this paper, we tend to present our practical approach that may solve the above challenges by using tcp multipath (MPTCP). With MPTCP, we divide every protocol stream into an information measure guarantee sub-band (BG) and a work-storage sub flow. We tend to treat all BG secondary flows and UDP flows as BG traffic and WC secondary flows as WC traffic. We tend to enable BG traffic to submit to a high priority queue on the switches to make sure the allotted bandwidth. WC traffic passes through a low priority queue to capture unused bandwidth without damaging the bandwidth guarantee. We have enforced our approach in conjunction with the implementation of the Linux MPTCP kernel.
[3] J. Guo, F. Liu, J. C. S. Lui, and H. Jin., proposed "Fair network bandwidth allocation in iaas data centers via a cooperative game approach". With a broad application of virtualization technology, tenants will access the rental of shared resources within the infrastructure data centers as a service (IaaS). The data center network that depends on the standard transport layer protocols suffers from injustice because of the shortage of bandwidth guarantees of the virtual machine (VM). During this paper, we tend to model the allocation of datacenter bandwidth as a cooperative game, to VM-based equity within the knowledge center with two main objectives: 1) to ensure bandwidth for virtual machines based on their bandwidth needs, and 2) share the residual bandwidth in proportion to the weights of virtual machines. We tend to propose a bandwidth allocation algorithmic rule, Falloc, to understand the asymmetric solution of Nash (NBS) negotiation within the networks of centers of data, that exactly meets our objectives. The cooperative structure of the algorithm is exploited to develop a web algorithm for practical implementation within the real world. we tend to valid Falloc with experiments in various scenarios and demonstrated that by adapting to the various virtual machine network requirements, Falloc are able to do fairness among the VMs and balance the compensation between the bandwidth guarantee and therefore the proportional bandwidth exchange. Our large-scale track-based simulations verify that Falloc achieves high usage whereas maintaining equity among VMs in data centers.

[4] T. Lam et al., proposed "NetShare: Virtualizing Bandwidth within the Cloud". There is still no efficient regulation mechanism for allocating bandwidth resources. The cloud platform sometimes has a worse performance network process that reduces the quality of services as a result of the bandwidth of resources cannot be adjusted flexibly. Therefore, this paper proposes a new mechanism of allocation and management of bandwidth resources, called Dynamic Virtualized bandwidth Allocation (DVBA), that provides management a centralized SDN / Open Flow design as a basis for virtualized bandwidth and even fixes insufficient static bandwidth and equitable allocation of NETSHARE resources. Therefore, the DVBA methodology that allocates bandwidth resources considers the status, quality of service, packet size and use of wide charge queues of band. Then, we are going to conduct simulations to match 
DVBA with NETSHARE using KPI in terms of performance and average response times. Finally, the experimental results indicate that DVBA has superior performance and higher average mean latency. In the future, we are going to conduct a lot of simulations with different KPIs, like packet loss rate and latency to demonstrate that DVBA's proposal will dynamically regulate bandwidth allocation and alter easy-to-regulate network resources.

\section{BANDWIDTH MEASUREMENTS}

\subsection{AVAILABLE BANDWIDTH}

A bandwidth measurement is made from the bandwidth capability of a link, from the end-to-end capability of a route, from the available bandwidth of a link and from the available bandwidth of a route. Each the measured available bandwidth and therefore the measured link capability rely on the scale of the probe package, the wide available bandwidth is beneficial as a result of it supports high-volume data transfers, short latencies, and high success rates. The bandwidth available for the application directly affects application performance. Network providers rent connections to customers and reload on the idea of the available bandwidth that is provided to customers. The available bandwidth is additionally a key concept in algorithms to avoid congestion and in intelligent routing systems.

- Path Capacity Bandwidth (PCB): Min_i (b_i)

- Min (b_i-c_i) where $c_{-} i$ is the cross traffic

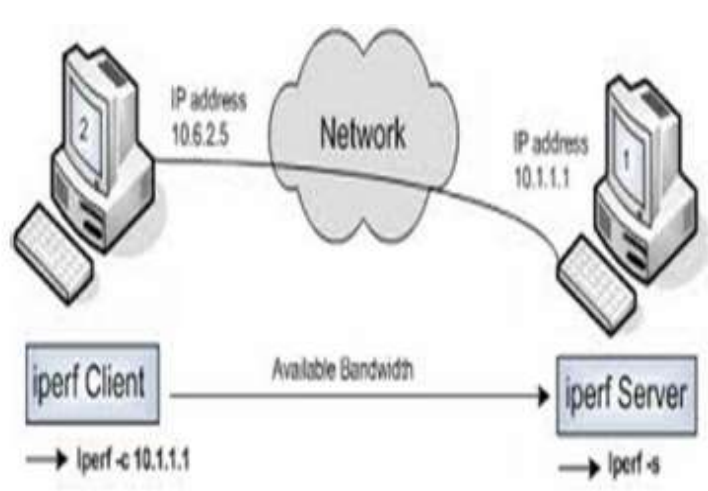

Fig: Available Bandwidth

\subsection{ESTIMATING AVAILABLE BANDWIDTH}

The techniques for estimating available bandwidth are divided into two main categories:

\section{* Passive measurement \\ * Active measurement}

\subsubsection{PASSIVE MEASUREMENT:}

Passive measurement is performed by observing the existing traffic without heavy the network. It is necessary to process the complete load on the link and requires access to all or any intermediate nodes within the network path to extract the end-to-end data. Monitor methods, like a simple network management protocol, which will offer elaborate statistics on network components, like capability and use of physical bandwidth; unfortunately, they require special access privileges that are typically not accessible to traditional users.

\subsubsection{ACTIVE MEASUREMENT}

Active measurement, on the other hand, directly analyses the properties of the network, generating the traffic required to perform the measurement. It is probably very efficient and accurate, its scope is restricted to the network routes that have recently transported user traffic. The packet pair technique is used within the active polling technique. It is a convenient way to estimate the network bandwidth available to traditional users because it does not need access to routers. Though active techniques inject additional traffic into the network path; it is more applicable to use active probe measurement to measure the available end-to-end bandwidth. The fundamental idea of packet pairs is that the sender sends a few of packets, that the destination returns to the echo. By measuring variations in packet spacing, the sender will estimate the bandwidth properties of the network path. Though the packet-pair mechanism could be a reliable methodology for measuring the capacity of a network route bottleneck capacity, its use to measure the available bandwidth has had a lot of varied results.

$$
\mathrm{BW}=\frac{\mathrm{f}_{\mathrm{o}}}{\mathrm{Q}} \ldots
$$

$$
B W=f_{2}-f_{1} \ldots(3)
$$


Where,

BW=Bandwidth of a circuit in units of frequency

$f_{0}=$ Center frequency

$f_{2}=$ Upper cut off frequency

$f_{1}=$ Lower cut-off frequency

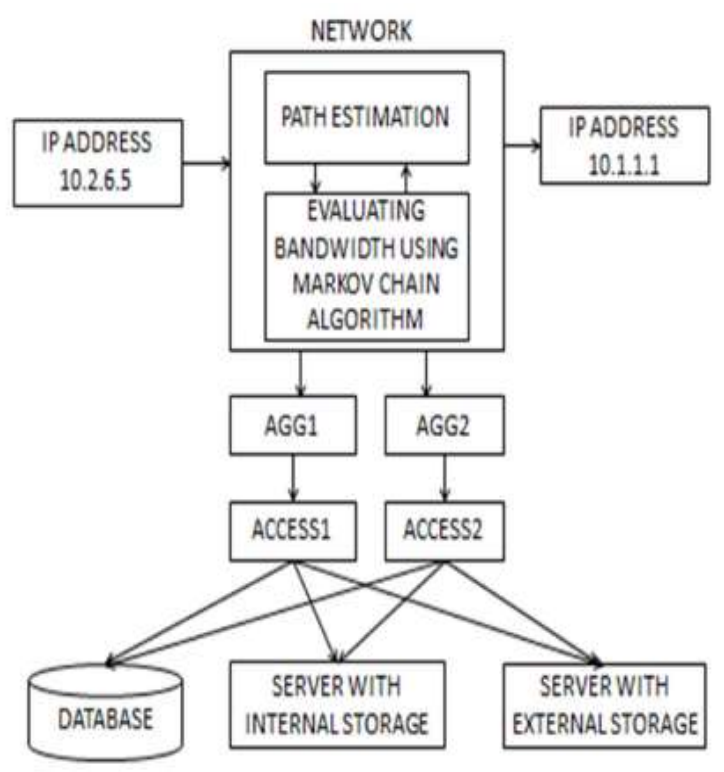

Figure: Architecture Diagram

\section{METHODOLOGY}

\subsection{NETWORK}

Client-server computing or networking may be a distributed application architecture that partitions tasks or workloads between service providers (servers) and service requesters, called clients. Typically clients and servers operate over a network on separate hardware. A server machine may be a high-performance host that is running one or additional server programs that share its resources with clients. A client also shares any of its resources; clients thus initiate communication sessions with servers that look (listen to) incoming requests.

\subsection{SUBPATH BANDWIDTH MEASUREMENT}

Two totally different measures used in end-to-end network bandwidth estimation are capacity bandwidth, or the maximum transmission rate that would be achieved between two hosts at the endpoints of a given path within the absence of any competing traffic, and available bandwidth, the portion of the capacity bandwidth on a path that would be acquired by a given flow at a given instant in time. Each of those measures is vital, and each captures totally different relevant properties of the network. Capacity bandwidth is a static baseline measure that applies over long time-scales (up to the time-scale at that network methods change), and is independent of the particular traffic dynamics at a time instant. Available bandwidth provides a dynamic measure of the load on a path, or more exactly, the residual capacity of a path.

\subsection{SHARED BANDWIDTH CAPACITY}

The type of applications which will be leveraged by the identification of shared capacity bandwidth we tend to consider scenarios illustrated. A client should select two out of three sources to use to transfer data in parallel. This situation could arise once downloading content in parallel from a set of mirror sites or multicast sources, or from a set of peer nodes in P2P environments. In the second situation, an overlay network should be found out between one source and two destinations.

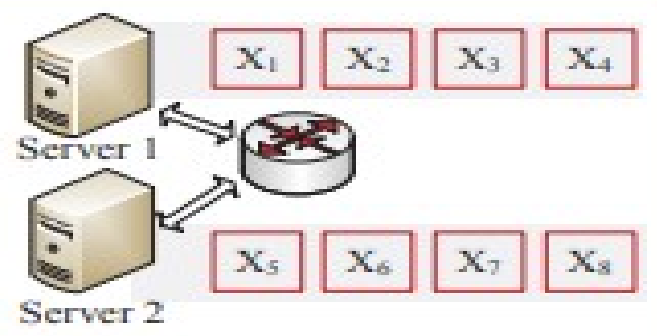

Fig. 3: Sharing network with traffic from server 2 to server 1.

\subsection{EVALUATING BANDWIDTH}

One will classify bandwidth estimation techniques into active and passive techniques. Active techniques, comprising most of the work in the literature, send probes for the only purpose of bandwidth activity. Passive techniques trust data packets for searching as exemplified in Lai and Baker's web timer tool that uses a packet-pair technique at the transport level to passively estimate capacity link bandwidth. The techniques we tend to propose during this paper are applied actively.

\section{CONCLUSION}

In this article we have presented an analysis and a comparative study of available bandwidth 
measurement techniques. We have shown measurements that illustrate the difference between bandwidth measurements. Previous work on bandwidth allocation for datacenters are not appropriate since rate limiting traffic is ineffective and causes system performance degradation. We propose Markov chain algorithm, the performance of this Markov chain algorithm is shown in terms of convergence, stability, fairness, queue length, link utilization, and packet loss ratio. The study presented in this paper was focused on the small number of criteria that seemed to be the most important. However, this study must be completed by considering other parameters and by evaluating the measurement.

\section{REFERENCE}

1 J. Lee et al., "Application-driven bandwidth guarantees in datacenters" in Proc. ACM SIGCOMM, 2014.

2 J. Guo et al., "On efficient bandwidth allocation for traffic variability in datacenters," in Proc. IEEE INFOCOM, Apr./May 2014.

3 A. Shieh, S. Kandula, A. Greenberg, C. Kim, and B. Saha, "Sharing the data center network," in Proc. 8th USENIX Conf. Netw. Syst. Design Implement., Mar. 2011.

4 C. Guo, G. Lu, H. Wang, S. Yang, C. Kong, P. Sun, W. Wu, and Y. Zhang, "Second net: A data center network virtualization architecture with bandwidth guarantees," in Proc. of ACM CoNEXT, 2010.

5 H. Rodrigues, J. Santos, Y. Turner, P. Soares, and D. Guedes, "Gatekeeper: Supporting bandwidth guarantees for multi-tenant datacenter networks," in Proc. of 3rd Workshop on I/O Virtualization. USENIX, 2011.

6 Z. Fang and B. Bensaou, "Fair bandwidth sharing algorithms based on game theory frameworks for wireless ad-hoc networks," in Proc. of IEEE INFOCOM, 2004.

7 H. Yä1che, R. Mazumdar, and C. Rosenberg, "A game theoretic framework for bandwidth allocation and pricing in broadband networks," IEEE/ACM Transactions on Networking (TON), vol. 8, no. 5, pp. 667-678, 2000.
8 R. Prasad, M. .Murray, C. Dovrolis, K. Claffy "Bandwidth Estimation: Metrics, Measurement Techniques, and Tools", IEEE Network, Nov-Dec 2003.

9 M. Jain, C. Dovrolis, "End-to-End Available Bandwidth: Measurement Methodology, Dynamics, and Relation with TCP Throughput". IEEE/ACM Transactions in Networking, August 2003.

10 A. Shriram, M. Murray, Y. Hyun, N. Brownlee, A. Broido, M. Fomenkov, K. Claffy " Comparison of Public End-to-End Bandwidth Estimation Tools on High-Speed Links", PAM'2005, Boston, March 2005.

11 C. Guo et al. "Second Net: A Data Center Network Virtualization Architecture with Bandwidth Guarantees". Technical Report MSRTR-2010-81, 2010.

12 N.Hu and P.Steenkiste, "Evaluation and characterization of available bandwidth probing techniques," IEEE Journal on Selected Areas in Communications, vol. 21, Aug. 2003.

13 Goldoni, E., Rossi, G., Torelli, A.” Assolo, a New Method for Available Bandwidth Estimation" Proceedings of the Fourth International Conference on Internet Monitoring ICIMP'09,May 2009.

14 Croce, D., Mellia, M., Leonardi, E. "The Quest for Bandwidth Estimation Techniques for large-scale Distributed Systems" Proceedings of ACM HotMetrics, 2009.

15 Lucian Popa et al., "Elastic Switch: practical work-conserving bandwidth guarantees for cloud computing”, ACM SIGCOMM 2013 conference on SIGCOMM, 2013.

16 J. Guo, F. Liu, J. C. S. Lui, and H. Jin, "Fair network bandwidth allocation in iaas datacenters via a cooperative game approach", IEEE/ACM Transactions on Networking (TON), Volume 24 Issue 2, April 2016.

17 Terry Lam and George Varghese, "Netshare: Virtualizing bandwidth within the cloud," UCSD, Tech. Rep., 2009. 